\title{
Properties of unique degree sequences of 3-uniform hypergraphs
}

\author{
M. Ascolese ${ }^{1}$, A. Frosini ${ }^{1}$, W.L. Kocay ${ }^{2}$, and L. Tarsissi ${ }^{3}$ \\ 1 Università di Firenze, Dipartimento di Sistemi e Informatica, Viale Morgagni 65, \\ 50134 Firenze, Italy corresponding author: andrea.frosini@unifi.it \\ 2 Department of Computer Science and St. Pauls College, University of Manitoba, \\ Winnipeg, Manitoba, CANADA \\ 3 LIGM, Univ Gustave Eiffel, CNRS, ESIEE Paris, F-77454 Marne-la-Vallée, France
}

\begin{abstract}
In 2018 Deza et al. proved the NP-completeness of deciding wether there exists a 3-uniform hypergraph compatible with a given degree sequence. A well known result of Erdös and Gallai (1960) shows that the same problem related to graphs can be solved in polynomial time. So, it becomes relevant to detect classes of uniform hypergraphs that are reconstructible in polynomial time. In particular, our study concerns 3 -uniform hypergraphs that are defined in the $N P$-completeness proof of Deza et al. Those hypergraphs are constructed starting from a nonincreasing sequence $s$ of integers and have very interesting properties. In particular, they are unique, i.e., there do not exist two non isomorphic 3 -uniform hypergraphs having the same degree sequence $d_{s}$. This property makes us conjecture that the reconstruction of these hypergraphs from their degree sequences can be done in polynomial time. So, we first generalize the computation of the $d_{s}$ degree sequences by Deza et al., and we show their uniqueness. We proceed by defining the equivalence classes of the integer sequences determining the same $d_{s}$ and we define a (minimal) representative. Then, we find the asymptotic growth rate of the maximal element of the representatives in terms of the length of the sequence, with the aim of generating and then reconstructing them. Finally, we show an example of a unique 3-uniform hypergraph similar to those defined by Deza et al. that does not admit a generating integer sequence $s$. The existence of this hypergraph makes us conjecture an extended generating algorithm for the sequences of Deza et al. to include a much wider class of unique 3-uniform hypergraphs. Further studies could also include strategies for the identification and reconstruction of those new sequences and hypergraphs.
\end{abstract}

AMS classification: 05C65, 05C60, 05C99

Keywords: Hypergraph · Graphic sequence - Uniqueness problem . Analysis of algorithms

\section{Introduction}

The notion of hypergraph naturally extends that of graphs, where each edge is defined to be a subset of the vertices (see [2] for basic definitions and results on hypergraphs). 
In this paper, we consider simple hypergraphs, i.e., hypergraphs that are loopless and with distinct edges. Here a loop is considered to be an edge consisting of just one vertex. A hypergraph is h-uniform if every edge is a subset of cardinality $h$.

The degree sequence of a simple hypergraph is the sequence of the degrees of its vertices arranged in non-increasing order. A degree sequence usually does not characterize the hypergraph it is related to, but however it reveals interesting properties. For this reason, degree sequences are widely studied in (hyper)graph theory. We say that a reconstruction of a degree sequence $d$ is a (hyper)graph whose degree sequence is $d$. One of the most challenging problems related to hypergraph degree sequences is the reconstruction of a compatible (hyper)graph, if any [4]. For graphs, this problem has been solved in a milestone paper by Erdös and Gallai [7], in 1960, providing an efficiently computable characterization of them. And an algorithm to construct a graph with a given degree sequence was given by Havel [10] and Hakimi [9]. On the other hand, the same problem for hypergraphs remained unsolved till 2018, when Deza et al. in [6] proved its NP-completeness, even for the simplest case of 3-uniform hypergraphs.

As a consequence, the study of wide classes of uniform hypergraphs whose reconstruction from a degree sequences can be performed in polynomial time has acquired more and more relevance, in order to spot the hard core of the generic reconstruction problem.

Recently, but still before the result in [6], some necessary conditions had been given for a sequence to be the degree sequence of an $h$-uniform hypergraph. Such a sequence is called an $h$-sequence. Most of these generalized the Erdös and Gallai theorem, or were based on two well known theorems by Havel [10] and Hakimi [9]. On the other hand, few necessary conditions were known. Among them, one of prominent interest is provided in [1], which uses Dewdney's theorem and sets a lower bound on the length of a sequence related to an $h$-uniform hypergraph. This result has been algorithmically rephrased in $[3,8]$.

The present study focuses on investigating the properties of the 3-sequences that originate from a generalization of the gadget used by Deza et al. in [6] for their NP-completeness proof. These are denoted by $\mathcal{D}_{n}$, according to their length $n$. The relevance of those sequences is mainly due to their uniqueness property, i.e., there exists a unique 3-hypergraph compatible with them, up to isomorphism. It is known that there exists an operator called a trade that allows one to travel amongst all hypergraphs having the same degree sequence [11]; here we prove that the 3 -hypergraphs related to the elements of each $\mathcal{D}_{n}$ act as a sort of fixed point for this operator.

Furthermore, since each element of $\mathcal{D}_{n}$ can be related to an infinite number of integer sequences, we group them into equivalence classes and we choose a representative for each class. We compute a lower bound to the asymptotic growth of the representative's elements gaining information about the cardinality of $\mathcal{D}_{n}$ and obtaining clues for a strategy for reconstruction. 
From this preliminary study, a series of open problems results, with the long term aim of characterizing and of reconstructing wider classes of unique degree sequences. An example is given in Example 2.

So, in the next section, we will provide definitions and the results useful for our study. Section 3 will be devoted to presenting the most relevant properties of the degree sequences in $\mathcal{D}_{n}$ and the asymptotic growth of the elements of their representatives. In Section 4, we consider the reconstruction problem of a simple subclass of $\mathcal{D}_{n}$ and the isomorphism problem on unique sequences. We conclude our work by pointing out some open questions in Section 5.

\section{Basic Notions and Definitions}

We recall the basic definition of hypergraphs and fix the notation used in the rest of the paper. A hypergraph $H$ is defined as a pair $H=(V, E)$ such that $V$ is the set of vertices and $E \subset \mathcal{P}(V) \backslash\{\emptyset\}$ is the set of hyperedges, or briefly edges when no ambiguities occur, where $\mathcal{P}(V)$ is the power set of $V$.

A hypergraph is simple if it is loopless and has distinct edges. In other words, it does not allow singleton edges or edges that are contained in or equal to other edges. The degree of a vertex $v \in V$ is the number of edges containing $v$. The degree sequence $d=\left(d_{1}, d_{2}, \ldots, d_{n}\right)$ of a hypergraph $H$ is the list of its vertex degrees usually arranged in non-increasing order. Let us denote by $\sigma(d)$ the sum of the elements of $d$. When $\mathcal{H}$ is $k$-uniform, the sequence $d$ is called $k$-graphic. Notice that the case $k=2$ corresponds to graphs, and a 2-graphic sequence is simply called graphic.

The seminal book by Berge [2] contains some essential results about hypergraphs and also information about their applications.

The problem of characterizing the graphic sequences of simple graphs was solved by Erdös and Gallai [7], in 1960, and algorithmically by Havel [10] and Hakimi [9], while only recently Deza et al. in [6] have shown the $N P$-completeness of the characterization of $k$-graphic sequences, with $k \geq 3$. The 3-Hypergraph Degree Sequence Problem is the question of determining whether a given sequence $d$ is the degree sequence of a 3-hypergraph.

In their proof, the authors mapped the instances of the known $N P$-complete problem 3-Partition into instances of the 3-Hypergraph Degree Sequence problem. The mapping has the property that there is a 1-to- 1 correspondence between the solutions of the instances $I$ of 3-partition and the 3-hypergraphs having the prescribed degree sequence $d$, computed from $I$.

To compute $d$, Deza et al. used an intermediate step in which they constructed a "gadget", and computed its degree sequence $d^{\prime}$ from $I$. This sequence turns out to have very interesting properties, as shown in the next sections, and it constitutes the focus of our research.

Hereafter, we define the procedure Gen-pi $(s)$ that generalizes the gadget computation presented in [6], regardless the specific characteristics of the length and element sum of the instance of 3-Partition. The input of this computation is an integer sequence $s=\left(s_{1}, \ldots, s_{n}\right)$, and it returns a degree sequence, denoted 
$d_{s}$, to emphasize its dependence on $s$, of a 3-hypergraph. Following the notation in [6], let $\{0,1\}_{3}^{n}$ be the set of all the binary sequences of length $n$ having exactly three elements equal to 1 and let $s^{T}$ be the transpose of the vector $s$.

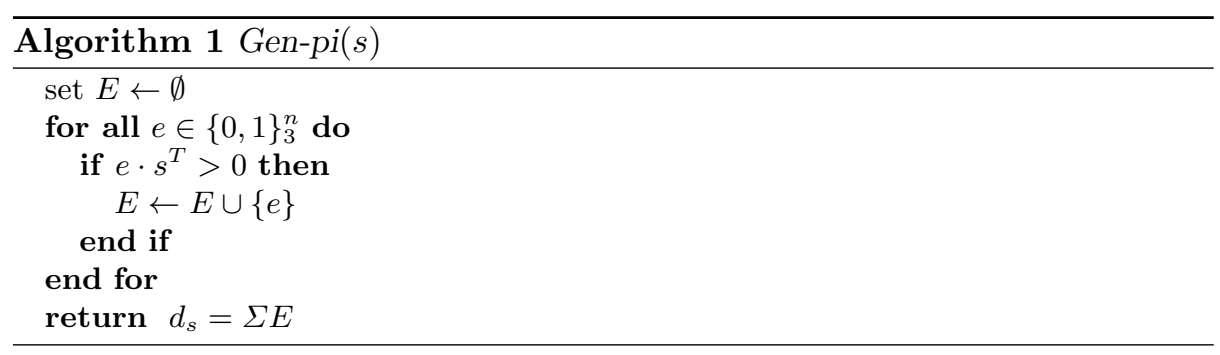

The class of degree sequences of length $n$ generated by the algorithm Gen-pi from an input sequence $s$ is indicated as $\mathcal{D}_{n}$.

The action of Gen-pi on $s$ will be clearer after introducing the notion of incidence matrix of a hypergraph. Given a hypergraph $H=(V, E)$ such that $\left|V_{\mathcal{H}}\right|=n$ and $\left|E_{\mathcal{H}}\right|=m$, its incidence matrix is a $m \times n$ binary matrix where $a_{i, j}=1$ if and only if the edge $e_{i}$ contains the vertex $v_{j}$, otherwise $a_{i, j}=0$. So $\sum_{i=1}^{m} a_{i, j}=d_{j}$ is the degree of the vertex $v_{j}$, and when $\mathcal{H}$ is $k$-uniform we have $\sum_{j=1}^{n} a_{i, j}=k$ for each edge $e_{i}$.

We observe that, for $k$-hypergraphs, the property of being simple means that all the rows of the incidence matrix are different.

Example 1 Let us consider the integer sequence $s=(3,2,0,-1,-2)$. The output of Gen-pi(s) is the degree sequence $d_{s}=(5,4,4,3,2)$. Figure 2 shows a 3-hypergraph $H\left(d_{s}\right)$ having $d_{s}$ as degree sequence, together with its related incidence matrix $M\left(d_{s}\right)$.

The matrix representation of the hypergraph $H\left(d_{s}\right)$ in Fig. 2 provides an immediate idea of the action of Gen-pi on $s$. In the sequel, we will consider only degree sequences obtained from sequences $s$ such that $s_{1}+s_{2}>s_{n}$, i.e., without null columns or, equivalently, hypergraphs without isolated vertices.

\section{Properties of the elements of $\mathcal{D}_{n}$}

A relevant property of a degree sequence $d_{s}$ directly follows from the action of Gen-pi on a generic integer sequence $s$ :

Theorem 1 There exists one only 3-hypergraph (up to isomorphism) having degree sequence $d_{s}$.

Proof. The result can be obtained by contradiction. Let $d_{s}=\left(d_{1}, \ldots, d_{n}\right)$ and $s=\left(s_{1}, \ldots, s_{n}\right)$ be as in the algorithm Gen-pi. We observe that the number $\operatorname{Max}=\sum_{i=1}^{n} s_{i} d_{i}$ is the maximum that can be realized by a sequence of 


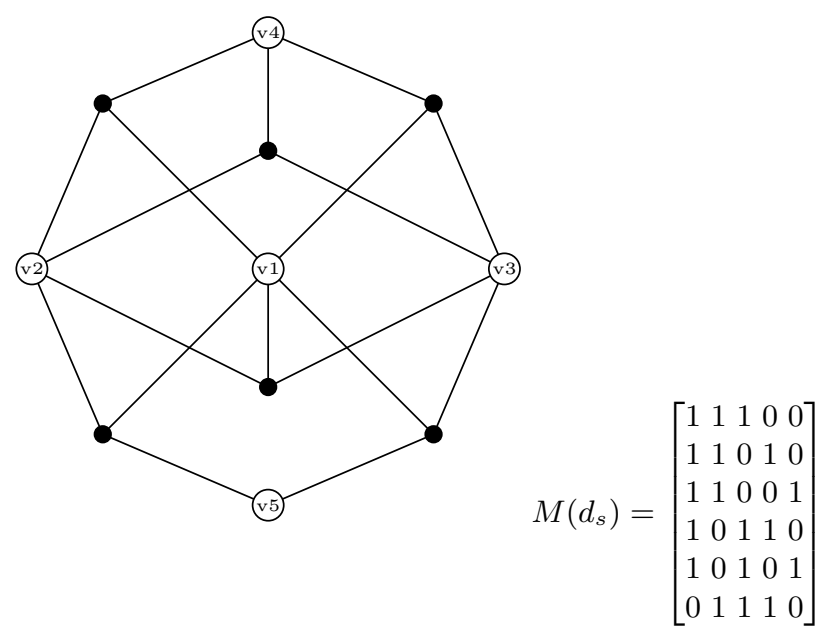

Fig. 1. The 3-hypergraph $H\left(d_{s}\right)$ and its related incidence matrix $M\left(d_{s}\right)$. The black nodes represent the edges of the 3-hypergraph and the white circles represent its vertices.

$m=\sum_{i=1}^{n} d_{s} / 3$ different triplets $\left(s_{i_{1}}, s_{j_{1}}, s_{k_{1}}\right), \ldots,\left(s_{i_{m}}, s_{j_{m}}, s_{k_{m}}\right)$ of elements of $s$, containing all the edges $\left(i_{1}, j_{1}, k_{1}\right), \ldots,\left(i_{m}, j_{m}, k_{m}\right)$ of the 3-hypergraph $H\left(d_{s}\right)$. Any other 3-hypergraph $H\left(d_{s}\right)^{\prime}$ having the same degree sequence would involve at least an edge $e=(i, j, k)$ not in $H\left(d_{s}\right)$, so that $s_{i}+s_{j}+s_{k}=t \leq 0$. As a consequence, the remaining $m-1$ different edges $\left(i_{1}^{\prime}, j_{1}^{\prime}, k_{1}^{\prime}\right), \ldots,\left(i_{m-1}^{\prime}, j_{m-1}^{\prime}, k_{m-1}^{\prime}\right)$ of $H\left(d_{s}\right)^{\prime}$ must satisfy $\Sigma_{z=1}^{m-1} s_{i_{z}^{\prime}}+s_{j_{z}^{\prime}}+s_{k_{z}^{\prime}}=$ Max $+t$, and we reach a contradiction.

We say that the sequence $d_{s}$ is unique, as well as the corresponding 3hypergraph.

Note that the number of elements of $\mathcal{D}_{n}$ is finite, since each $d_{s}$ has $\left(\begin{array}{l}n \\ 3\end{array}\right) \frac{3}{n}$ as a maximum entry. So, by cardinality reasons, there exist an infinite number of non-increasing integer sequences $s$ that generate at least one degree sequence $d_{s}$. An easy check reveals that for each degree sequence $d_{s}$ there exists an infinite number of generating integer sequences.

As an example, all the length $n$ sequences of positive integers generate the same constant degree sequence $d_{s} \in \mathcal{D}_{n}$ whose elements are $\left(\begin{array}{l}n \\ 3\end{array}\right) \frac{3}{n}=\frac{(n-1)(n-2)}{2}$. Obviously, this sequence is maximal in $\mathcal{D}_{n}$ w.r.t the lexicographical order.

An easy check reveals that if a sequence $s$ has two equal elements $s_{i}$ and $s_{i+1}$, then also the elements $d_{i}$ and $d_{i+1}$ of the related $d_{s}$ degree sequence are equal. The reverse is also true.

Property 1 If there exists an index $i<n$ of $d_{s}$ such that $d_{i}=d_{i+1}$, then there exists a sequence $s^{\prime}$ such that $s_{i}^{\prime}=s_{i+1}^{\prime}$ and $d_{s}=d_{s^{\prime}}$. 
Proof. Let us construct $s^{\prime}$ from $s$ : if $s_{i}=s_{i+1}$, then $s^{\prime}=s$. So, let us consider the case $s_{i} \neq s_{i+1}$. Since $s_{i}>s_{i+1}$, then each triplet $s_{i+1}+s_{j}+s_{k}>0$ implies the triplet $s_{i}+s_{j}+s_{k}>0$, and each triplet $s_{i}+s_{j^{\prime}}+s_{k^{\prime}} \leq 0$ implies the triplet $s_{i+1}+s_{j^{\prime}}+s_{k^{\prime}} \leq 0$, with $j, j^{\prime}, k, k^{\prime} \leq n$. Since $d_{i}=d_{i+1}$, then the reverse of both implications also holds, and furthermore, these inequalities also hold if $s_{i}$ is replaced by an integer $s_{i+1} \leq \bar{s} \leq s_{i}$. For the inequalities that involve both $s_{i}$ and $s_{i+1}$, i.e., those of the forms $s_{i}+s_{i+1}+s_{k}>0$ or $s_{i}+s_{i+1}+s_{k^{\prime}} \leq 0$, they are preserved when $s_{i}$ and $s_{i+1}$ are both replaced by the value $\bar{s}=\frac{s_{i}+s_{i+1}}{2}$. Unfortunately, $\bar{s}$ is not always an integer, and a final observation is required: the inequalities used in the Gen-pi procedure are preserved when all the elements of $s$ are doubled, i.e., by abuse of notation, it holds $d_{s}=d_{2 * s}$.

Putting things together, from $s$ we can define the sequence $s^{\prime}$ as follows: first we initialize $s^{\prime}=2 s$, then we set $s_{i}^{\prime}=s_{i+1}^{\prime}=\frac{s_{i}^{\prime}+s_{i+1}^{\prime}}{2}=\bar{s}^{\prime}$. Doubling the elements of $s$, we find that $\bar{s}^{\prime}$ is integer, and since $s_{i}^{\prime} \leq \bar{s}^{\prime} \leq s_{i+1}^{\prime}$, all the inequalities that define $d_{s}$ are preserved, as required.

Property 2 Let $s_{i}=s_{i+1}$ be two elements of an integer sequence $s$. The $i^{\text {th }}$ column and the $(i+1)^{\mathrm{st}}$ column of the incidence matrix $M_{s}$ of the hypergraph $H_{s}$ generated by procedure Gen-pi(s) are equal.

The proof directly follows from the fact that the elements $d_{i}$ and $d_{i+1}$ satisfy the same inequalities, and so they are present in the edges involving the same vertices.

Returning to the equivalence classes of the elements in $\mathcal{D}_{n}$, we propose as representative of the (non-void) class $[d]$, the sequence $s_{d}$ that is minimal in lexicographic order. We stress the fact that $s_{d}$ has the minimal first element among all the elements of $[d]$.

Unfortunately, equal elements of $d$ do not always correspond to equal elements in the representative of $s_{d}$. As an example, exhaustive computation reveals that the representative of $d=(6,5,5,4,4)$ is $s_{d}=(2,1,1,0,-1)$.

In order to compute and characterize the representative $s_{d}$ of each equivalence class $[d]$, it is useful to understand the growth rate of the elements inside $s_{d}$ according to length, with special attention to its first (and maximal) one.

So, for each length $n$ and each $d \in \mathcal{D}_{n}$, we denote

$$
M_{n}=\max _{s_{d}}\left\{s_{1}: s_{d}=\left(s_{1}, s_{2}, \ldots, s_{n}\right)\right\} .
$$

We call the sequences $s_{d}$ where such a maximal first element is present maximal sequences. The first elements of the sequence $\left\{M_{n}\right\}_{n>2}$ up to $n=8$, obtained by exhaustive computation are 1,1,2,4,6,10. For each $n$, there are several degree sequences whose representatives have the same maximal first element. The following table shows some of them according to the length parameter $n$ :

Let us investigate the asymptotic growth of the sequence $\left\{M_{n}\right\}_{n>3}$ by constructing a class of degree sequences $\mathcal{C}$ that provides a lower bound to it. 


\begin{tabular}{|c|c||c|c|}
\multicolumn{2}{|c||}{$n=5$} & \multicolumn{2}{|c|}{$n=7$} \\
\hline$s$ & $d_{s}$ & $s$ & $d_{s}$ \\
\hline$[2,1,1,0,-1]$ & {$[6,5,5,4,4]$} & {$[6,3,2,0,-1,-2,-3]$} & {$[15,11,10,9,8,7,6]$} \\
{$[2,1,1,-1,-2]$} & {$[5,4,4,3,2]$} & {$[6,3,2,0,-2,-3,-4]$} & {$[13,10,9,7,6,5,4]$} \\
{$[2,1,0,0,-1]$} & {$[6,4,4,4,3]$} & {$[6,3,1,1,0,-2,-3]$} & {$[15,13,10,10,9,8,7]$} \\
{$[2,1,0,-1,-2]$} & {$[4,3,2,2,1]$} & {$[6,2,1,1,-1,-2,-3]$} & {$[15,10,9,9,8,7,5]$} \\
{$[2,0,0,-1,-1]$} & {$[5,3,3,2,2]$} & {$[6,2,1,0,-1,-2,-3]$} & {$[15,9,8,7,7,6,5]$} \\
\hline
\end{tabular}

\begin{tabular}{|c|c||c|c|}
\multicolumn{2}{|c||}{$n=6$} & \multicolumn{2}{c|}{$n=8$} \\
\hline$s$ & $d_{s}$ & $s$ & $d_{s}$ \\
\hline
\end{tabular}

$[4,2,1,0,-1,-2][10,8,7,6,6,5][[10,4,3,2,0,-1,-3,-6][21,16,15,14,13,12,10,7]$

$[4,1,1,-1,-1,-2][[10,6,6,5,5,4]|[10,4,2,2,1,-2,-4,-5]|[21,17,14,14,13,12,9,8]$

Table 1. Table of the representatives $s$ of the equivalence classes $\left[d_{s}\right]$ having maximal first element for the sequence lengths $n=5,6,7$ and 8 .

First we define the operator Extend that allow us to compute from a sequence $s=\left(s_{1}, \ldots, s_{n}\right)$ a sequence $s^{\prime}=\left(s_{1}^{\prime}, \ldots, s_{n+2}^{\prime}\right)=\operatorname{Extend}(s)$ as follows:

$$
s_{i}^{\prime}=\left\{\begin{array}{lr}
s_{i-1}, & \text { for } 2 \leq i \leq n+1 \\
s_{1}+s_{2}-s_{n}+1, & \text { for } i=1 \\
-\left(s_{1}+s_{2}\right), & \text { for } i=n+2
\end{array}\right.
$$

The following property holds

Property 3 If $s$ is the representative of the class $[d] \in D_{n}$, then $s^{\prime}=\operatorname{Extend}(s)$ is the representative of the respective class in $D_{n+2}$.

Proof. Let $d_{s^{\prime}}$ be the degree sequence generated by $s^{\prime}$. We proceed by contradiction assuming there exists a representative $t=\left(t_{1}, \ldots, t_{n+2}\right)$ of $\left[d_{s^{\prime}}\right]$ different from $s^{\prime}$ and having $t_{1}<s_{1}^{\prime}$. We notice that the sequence $\tilde{t}=\left(t_{2}, \ldots, t_{n+1}\right)$ generates $d$, since the elements $s_{2}^{\prime}+s_{3}^{\prime} \leq s_{n+2}^{\prime}$, and such inequality must hold also in $t$, so also the elements $t_{2}, \ldots, t_{n+1}$ are not involved in any edge including $t_{n+2}$. On the other hand, by construction, $s_{2}^{\prime}+s_{3}^{\prime}=-s_{n+2}^{\prime}$, so it holds $t_{n+2} \leq s_{n+2}^{\prime}$.

Finally, we notice that the inequality $s_{1}^{\prime}+s_{n+1}^{\prime}+s_{n+2}^{\prime}>0$ also holds since $s_{1}^{\prime}+s_{n+1}^{\prime}+s_{n+2}^{\prime}=1$. In order to preserve the same inequality in $t$, i.e., $t_{1}+t_{n+1}+$ $t_{n+2}>0$, having $t_{n+2} \leq s_{n+2}^{\prime}$, we need $t_{1} \geq s_{1}^{\prime}$, and we reach a contradiction.

We underline that by iterating the application of the procedure Extend to a sequence $s$ of length $n$, it produces longer sequences having the same length parity as $s$.

To illustrate the action of Extend, we depict its behaviour on the incidence matrices $H_{s}$ and $H_{s^{\prime}}$, with $s^{\prime}=\operatorname{Extend}(s)$. The basic idea is to extend $H_{s}$ by adding an initial and a final column, as well as a set of starting rows, maximizing their number while leaving those in $H_{s}$ unchanged: 


$$
H_{s^{\prime}}=\left(\begin{array}{ccccccc}
1 & 1 & 1 & 0 & \ldots & \ldots & 0 \\
1 & 1 & 0 & 1 & \ldots & \ldots & 0 \\
\vdots & & & & & & \\
1 & \ldots & \ldots & \ldots & \ldots & 1 & 1 \\
0 & & & & & & 0 \\
\vdots & & & H_{s} & & \\
0 & & & & & & 0 \\
& & & & & &
\end{array}\right)
$$

Let $s_{0}=\left(s_{0,1}, \ldots, s_{0, n}\right)$ be an integer sequence, and $s_{k}=\left(s_{k, 1}, \ldots, s_{k, n+2 k}\right)=$ $\operatorname{Extend}^{k}\left(s_{0}\right)$ be the sequence obtained by recursively applying the procedure Extend $k$-times to $s_{0}$. The following result holds

Theorem 2 The integer sequence $\left\{s_{k, 1}\right\}_{k}$ satisfies the recurrence relation:

$$
s_{k, 1}=s_{k-1,1}+2 s_{k-2,1}+s_{k-3,1}+1 \quad k \geq 3
$$

with $s_{1,1}$ and $s_{2,1}$ being the first elements of $s_{1}=\operatorname{Extend}\left(s_{0}\right)$, and $s_{2}=\operatorname{Extend}^{2}\left(s_{0}\right)$, respectively.

Proof. The result immediately follows from the definition of the Extend operator:

$$
\begin{aligned}
& s_{k, 1}=s_{k-1,1}+s_{k-1,2}-s_{k-1, n+2(k-1)}+1 \\
& s_{k-1,2}=s_{k-2,1} \\
& s_{k-1, n+2(k-1)}=-s_{k-2,1}-s_{k-2,2}
\end{aligned}
$$

Replacing $s_{k-1,2}$ and $s_{k-1, n+2(k-1)}$ in the first equation we obtain the recurrence relation.

We observe that, starting from two representative sequences $s_{e}=(1,1,-1,-1)$ and $s_{o}=(2,1,0,-1,-2)$, having even and odd length, respectively, that are maximal w.r.t. the first element, the procedure Extend produces the two sequences Extend $\left(s_{e}\right)=(4,1,1,-1,-1,-2)$ and $\operatorname{Extend}\left(s_{o}\right)=(6,2,1,0,-1,-2,-3)$ that turn out to be two representatives with maximal first element of length 6 and 7 , respectively. This property is not maintained when considering $\operatorname{Extend}^{2}\left(s_{e}\right)=$ $(8,4,1,1,-1,-1,-2,-5)$, since $M_{8}=10$.

From this simple observation, we realize that the action of Extend on representatives with maximal first (and second) element deserves a deeper investigation in order to find an operator that allows us to pass from maximal representatives to maximal representatives. However, the Extend operator provides a lower bound to the growth rate of the $M_{n}$ sequence.

Theorem 3 The growth constant $\lambda$ of the sequence $\left\{s_{k, 1}\right\}_{k}$ is $2.147<\lambda<$ 2.148 . 
Proof. The result follows from constructing the generating function for the recurrence relation (1) whose denominator is $q(x)=\left(1-x-2 x^{2}-x^{3}\right)(1-x)$. The denominator has a unique minimal real root which can be computed numerically, $\rho=0.466$. Therefore the asymptotic behaviour is controlled by $\lambda$ that is the inverse of $\rho$, namely $f_{3, k} \sim(\lambda)^{k}$

Corollary 1 The growth constant $\lambda^{\prime}$ of $\left\{M_{n}\right\}_{n>3}$ is $\lambda^{\prime} \geq \lambda$.

\section{Further Results on Unique Sequences}

The property that each element $d$ of $\mathcal{D}_{n}$ is unique, has a great relevance in the reconstruction of the related 3-hypergraph $H_{d}$. In particular, it is remarkable that, once one detects some edges of $H_{d}$, then those edges will not be modified till the end of the reconstruction or, equivalently, they will not be involved in possible backtracking steps, so limiting the complexity of the process.

As an example, this is the case of one or more elements of $d=\left(d_{1}, \ldots, d_{n}\right)$ that are maximal or minimal. Focusing on the first element $d_{1}$, if its value is

$\left(\begin{array}{c}n-1 \\ 2\end{array}\right)$ this means that all edges involving the vertex $v_{1}$ are present, so they can be added and the process can proceed recursively on the next elements of $d$. Equivalently if $d_{1}=n-2$, then, by the definition of $d$, the edges involving $v_{1}$ are $\left(v_{1}, v_{2}, v_{3}\right),\left(v_{1}, v_{2}, v_{4}\right), \ldots,\left(v_{1}, v_{2}, v_{n}\right)$, that can be added to $H_{d}$.

Apart from those simple cases, some more are possible that may involve all the elements of $d$ in a complex pattern of entanglements. In the intent of discovering a polynomial time way of managing these fixed patterns, a first and natural reconstruction algorithm is provided to deal with those simple cases that we address as maximal instances. This name is due to the fact that the sequence is maximal w.r.t. the lexicographical order among those having the same sum of elements.

The algorithm Rec-max, here not fully detailed for brevity sake, accepts a degree sequence $d$ as input and produces the incidence matrix of a 3-hypergraph compatible with $d$, if $d$ is a maximal sequence, otherwise it fails. We use the notation $M \oplus h$ to append the row $h$ to the matrix $M$ and $\operatorname{deg}(M)$ to denote the vector of column sums of the matrix $M$, i.e., the degree sequence of the related hypergraph.

The proof of the correctness of the algorithm is straightforward.

\subsection{On the isomorphism properties of $\mathcal{D}_{n}$}

The remarkable properties of the elements of $\mathcal{D}_{n}$ have an interesting consequence on the isomorphism problem restricted to the class. We recall that two (hyper)graphs $H_{1}$ and $H_{2}$ are isomorphic if and only if one can pass from the incidence matrix of $H_{1}$ to that of $H_{2}$ by a first round of column shifts, that corresponds to a mapping $\varphi$ of the vertices of $H_{1}$ into those of $H_{2}$, followed by a second round of row shifts that allows to check the exact correspondence of the (hyper)edges. 


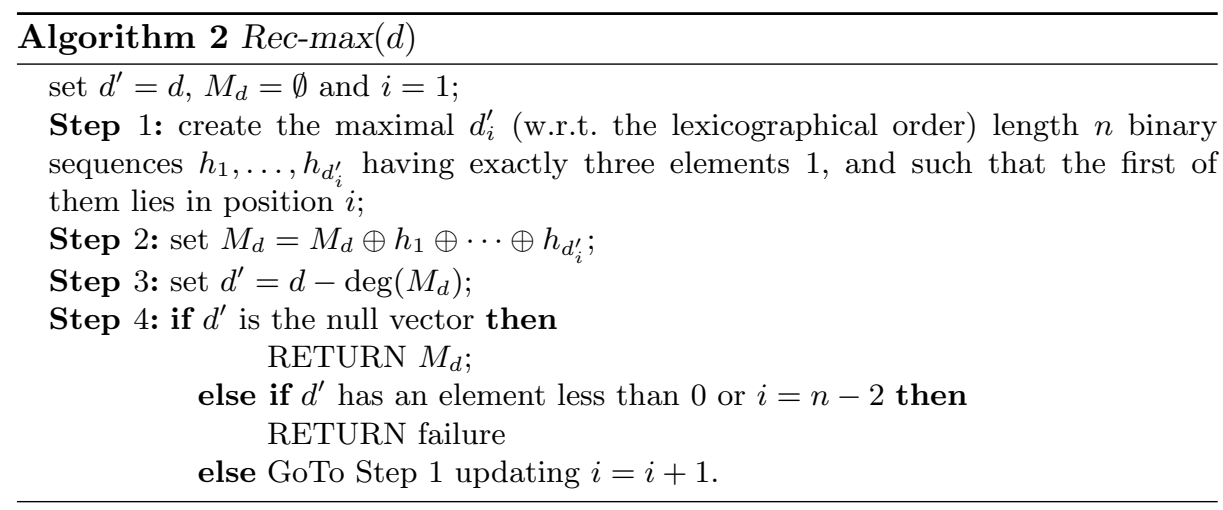

Obviously, to check the isomorphism, $\varphi$ has to preserve the vertices' degrees in $H_{1}$ and $H_{2}$, so in case of degree sequences with no equal elements $\varphi$ equals $I d$, the identity mapping.

Actually, in case of equal elements in the degree sequence of $H_{1}$ and $H_{2}, \varphi$ has, in general, to inspect the isomorphism for each possible permutations of the related columns (at present, no better strategy is available).

On the other hand, Property 2 states that, by construction, in each element $H \in \mathcal{D}_{n}$, the columns related to vertices having the same degree are equal, so again $\varphi=I d$ is a suitable mapping. This equality does not hold, in general, for each unique degree sequence (e.g., the degree sequence $d=(1,1,1,1,1,1)$ is unique w.r.t. 3-hypergraphs, but the check of the isomorphism of two related 3-hypergraphs $H_{1}$ and $H_{2}$ needs, in general, $\varphi$ to be different from $I d$ ).

The following example reveals a new potential research line concerning the characterization and reconstruction of unique sequences that include, but are not restricted to, those generated by Gen-pi:

Example 2 Consider the degree sequence $\hat{d}=(25,19,19,16,16,12,10,10,5)$. Its uniqueness is witnessed by the related matrix $M_{\hat{d}}$ in Fig. 2, and whose construction seems to be not so far from that performed by Gen-pi. It remains an open problem to find a suitable meaning to the words "not so far" that could lead to a generalization of Gen-pi.

The following computations show that there does not exist an integer sequence $s$ such that $\hat{d}=\hat{d}_{s}$. Recall that, by Property 1 , among all the sequences in $[\hat{d}]$, if any, there exists one $s$ such that $s_{2}=s_{3}, s_{4}=s_{5}$ and $s_{7}=s_{8}$ :

By the inequalities of Table 2 we obtain the result, in particular: by (1) and (3) we obtain $-2 s_{8}+s_{6}+s_{9}>0$, by this last and (2) we obtain $2 s_{2}+2 s_{5}+s_{6}+s_{9}>0$ and finally using (5) we reach $2 s_{2}+s_{9}>0$, against (4). 


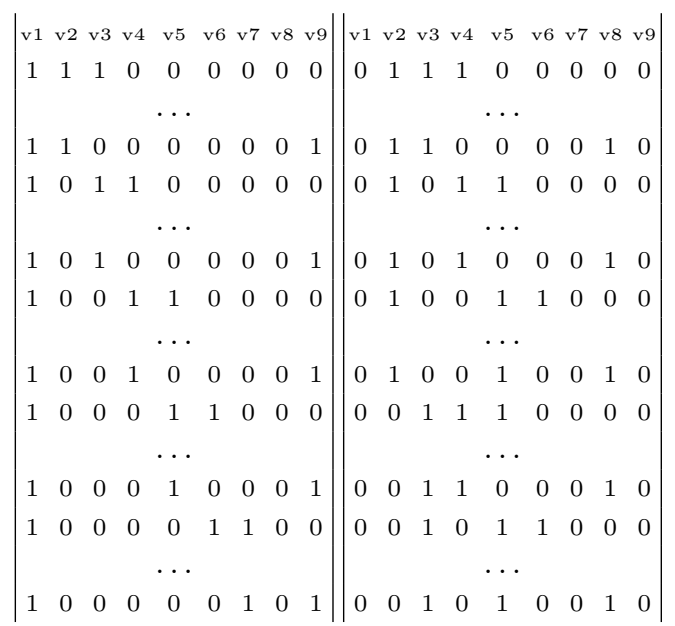

Fig. 2. The incidence matrix $M(\hat{d})$ of a 3-hypergraph having degree sequence $\hat{d}$. On the left the part of the matrix related to the edges involving $v_{1}$, while on the right the remaining ones. The 3-hypergraph is unique as can be seen by the construction of the matrix.

$(1,6,9) \in H_{\hat{d}} \rightarrow s_{1}+s_{6}+s_{9}>0(1)$

$(2,5,8) \in H_{\hat{d}} \rightarrow s_{2}+s_{5}+s_{8}>0(2)$

$(1,7,8) \notin H_{\hat{d}} \rightarrow s_{1}+2 s_{8} \leq 0$

$(2,3,9) \notin H_{\hat{d}} \rightarrow 2 s_{2}+s_{9} \leq 0$

$(4,5,6) \notin H_{\hat{d}} \rightarrow 2 s_{5}+s_{6} \leq 0$

Table 2. Inequalities related to some rows of the matrix $H_{\hat{d}}$. Edges are represented by triplets of vertex indices.

\section{Conclusions and Open Problems}

In this article, we consider the class $\mathcal{D}_{n}$ of degree sequences of 3-hypergraphs on $n$ vertices that extend those defined in [6] and that are computed starting from a given integer sequence. First, we prove that each degree sequence $d \in \mathcal{D}_{n}$ is unique, i.e., the related 3-hypergraph $H_{d}$ is unique up to isomorphism, then we define the representative integer sequence $s_{d}$ which leads to the reconstruction of $H_{d}$. Some properties of $s_{d}$ are shown, in particular we determine a lower bound to the growth rate of their maximal elements according to the length $n$, related to the number of edges of the 3-hypergraph $H_{d}$. This result is useful to generate and enumerate the elements of $\mathcal{D}_{n}$, establishing the size of the class. In this context, we point out two open problems:

i) define a variant of the Extend operator that allows to maintain the maximality property of the representatives;

ii) find the growth rate of the sequence $\left\{M_{n}\right\}_{n>2}$ and characterize the maximal representatives of each $\mathcal{D}_{n}$. 
Furthermore, a simple algorithm is defined to reconstruct the maximal (in lexicographic order) sequences of $\mathcal{D}_{n}$ having prescribed sum of elements. From its definition, we realize that a generalization to include all the elements of $\mathcal{D}_{n}$ would involve some backtracking when elements less than zero appear in $d^{\prime}$. We propose the following research line:

iii) find some properties related to the computation of $d_{s}$ from $s$ that prevent or, at least, restrict the backtracking in Rec-max. This will lead to the solution of the reconstruction problem related to $\mathcal{D}_{n}$. As an alternative, prove that it cannot be done in polynomial time.

Finally, the degree sequence $\hat{d}$ in Example 2 deserves attention: it admits a unique 3-hypergraph whose structure is close to that of the hypergraphs related to the elements of $\mathcal{D}_{n}$, but without being generated by an integer sequence. A final open problem is proposed:

$i v$ ) define a notion of structure of the elements of $\mathcal{D}_{n}$ and expand the class including unique degree sequences with similar structure. Investigate the properties of the new class.

\section{References}

1. Behrens, S., Erbes, C., Ferrara, M., Hartke, S.G., Reiniger, B., Spinoza, H., Tomlinson, C.: New Results on Degree Sequences of Uniform Hypergraphs, Electron. J. Comb., 20(4), \# P14 (2013).

2. Berge, C.: Hypergraphs, North-Holland, Amsterdam, (1989).

3. Brlek, S., Frosini, A.: A Tomographical Interpretation of a Sufficient Condition for h-Graphical Sequences DGCI 2016, Lect. Notes in Comput. Sc., 9647, pp.105 - 116 (2016).

4. Colbourne, C.J., Kocay, W.L. , Stinson, D.R.: Some NP-complete problems for hypergraph degree sequences Discrete Appl. Math., 14, pp.239 - 254 (1986).

5. Dewdney, A.K., Degree sequences in complexes and hypergraphs, Proc. Amer. Math. Soc., 53(2), pp.535 - 540 (1975).

6. Deza, A., Levin, A., Meesum, S.M., Onn, S.: Optimization over degree sequences, SIAM J. Disc. Math. 32(3), pp.2067 - 2079 (2018).

7. Erdös, P., Gallai, T.: Graphs with prescribed degrees of vertices (in Hungarian), Math. Lapok. 11 pp.264 - 274 (1960).

8. Frosini, A., Picouleau, C., Rinaldi, S.: On the degree sequences of uniform hypergraphs Lect. Notes in Comput. Sc., Vol. 7749, $300-311$ (2013).

9. Hakimi, S.: On realizability of a set of integers as degrees of the vertices of a lineargraph, I.J. Soc. Indust. Appl. Math., 10, pp.496-506 (1962).

10. Havel, V.: A remark on the existence of finite graphs (in Czech), Časopis. Pěst. Mat., 80, pp.477-480 (1955).

11. Kocay, W., Li, P.C.:On 3-Hypergraphs with Equal Degree Sequences, Ars Combinatoria, 82, pp.145 - 157 (2006) 